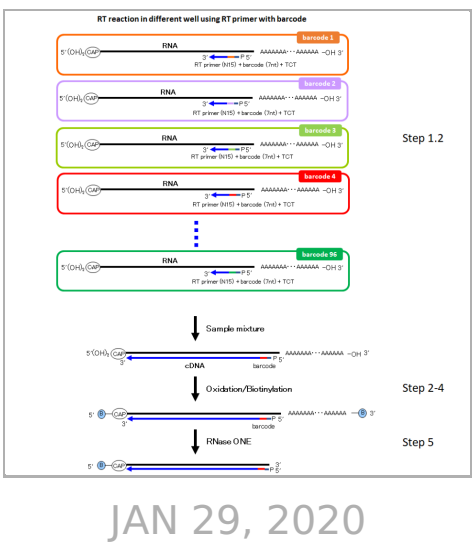

open ठAccess

DOI:

dx.doi.org/10.17504/protocol s.io. bbwkipcw

Protocol Citation: Hazuki Takahashi, Hiromi NishiyoriSueki, Piero Carninci 2020. Low Quantity single strand CAGE protocol. protocols.io https://dx.doi.org/10.17504/p rotocols.io. bbwkipcw

License: This is an open access protocol distributed under the terms of the Creative Commons Attribution License, which permits unrestricted use, distribution, and reproduction in any medium, provided the original author and source are credited

Protocol status: Working We use this protocol in our group and it is working.

Created: Jan 29, 2020

Last Modified: Jan 29, 2020

PROTOCOL integer ID: 32428

Keywords: CAGE (Cap Analysis of Gene Expression), Transcription Starting Sites, Gene promoter, enhancer RNA

\section{(3) Low Quantity single strand CAGE protocol}

$\rightarrow$ In 1 collection

\section{Hazuki Takahashi ${ }^{1}$, Hiromi Nishiyori-Sueki $^{1}, \quad$ Piero Carninci ${ }^{1}$ \\ ${ }^{1}$ Laboratory for Transcriptome Technology, RIKEN Center for Integrative Medical Sciences, Yokohama, Kanagawa 230-0045, Japan}

Hazuki Takahashi

Laboratory for Transcriptome Technology, RIKEN Center for In...

\section{ABSTRACT}

The $\underline{\text { Cap }}$ Analysis of Gene Expression (CAGE) is a powerful method to identify the Transcription Starting Sites (TSSs) of capped RNAs while simultaneously measuring transcript level. CAGE allows mapping at single nucleotide resolution all active promoters and enhancers. Large CAGE datasets have been over the years by individual labs and consortia, including the Encyclopedia of DNA Elements (ENCODE) and Functional Annotation Of the Mammalian Genome (FANTOM). These dataset constitute open resource for TSS annotations and gene expression analysis.

Here we provide a protocol for the most recent CAGE version of the method, which is called Low Quantity (LQ) single strand (ss) CAGE (LQ-)ssCAGE. The methods allow to profile samples starting from low quantity of RNAs. Samples may typically include cells cultured in small volumes to maximizing large scale perturbations, cellular compartments like nuclear RNAs or samples from developmental stages. The advantage of (LQ-)ssCAGE is to allow working with small samples and simultaneously avoiding PCR amplification: this step help to preserve exquisite quantification of expression. The protocol makes use of simplified steps, by loading single stranded cap selected cDNA. Further, is allows reducing the RNA amount down to $25 \mathrm{ng}$ and to shorten preparation time (less than 3 days) and to prepare libraries parallel, increasing the number of samples that one operator can reasonably handle (up to 96 samples). By proper organization of the flow of libraries preparation, an operator could further scale up the throughput. The obtained libraries are suitable for sequencing by various Illumina sequencing platforms using indexed reads and original barcode identifiers.

\section{MATERIALS}

\section{<Materials and Reagents>}

Product name

Maker

Cat. No.

Agencourt AMPure XP

BECKMAN COULTER 
A63881

Agencourt RNAClean XP

BECKMAN COULTER

A63987

KAPA Library Quantification Kits (500 reactions)-

KAPA BIOSYSTEMS

KK4835

Illumina / ABI Prism ${ }^{\circledR}$

Sodium periodate ACS Reagent Grade,

MP Biomedicals

0215257725-25 g

$5 \mathrm{M}$ Sodium Chloride, Molecular Biology Grade,

Promega

V4221

RNase One Ribonuclease,

Promega

M4261

Lithium chloride solution $8 \mathrm{M}$, for molecular biology, $\geq 99 \%$ SIGMA-ALDRICH

L7026-100ML

Sodium acetate buffer solution,

BioXtra, pH 7.0 $\pm 0.05\left(25^{\circ} \mathrm{C}\right)$, for molecular biology, $3 \mathrm{M}$, SIGMA-ALDRICH S2404-100ML

non-sterile; $0.2 \mu \mathrm{m}$ filtered,

Sodium periodate ACS Reagent Grade,

311448-5G

DNA Ligation Kit < Mighty Mix > ,

6023

Ribonuclease $\mathrm{H}$ (RNase $\mathrm{H})(20 \sim 60 \mathrm{U} / \mu \mathrm{L})$,

2150 A

10 mM dNTP Mix,

18427013

Dynabeads M-270 Streptavidin, 65305

Ribonuclease $\mathrm{H}(2 \mathrm{U} / \mu \mathrm{L})$, 18021014

RNaseZap RNase Decontamination Solution,

SIGMA-ALDRICH AM9780

SuperScript III Reverse Transcriptase,

TAKARA BIO INC.

18080044

UltraPure 0.5 M EDTA, pH 8.0,

15575020

UltraPure DNase/RNase-Free Distilled Water,

SCIENTIFIC 10977015

Biotin (Long Arm) Hydrazide,

TAKARA BIO INC.

SP-1100

0.5M EDTA (pH 8.0),

ThermoFisher SCIENTIFIC

ThermoFisher SCIENTIFIC

ThermoFisher SCIENTIFIC

ThermoFisher SCIENTIFIC

ThermoFisher SCIENTIFIC

Industries, 311-90075

ThermoFisher SCIENTIFIC

ThermoFisher

Vector Laboratories

10w/v\% Polyoxyethylene(20) Sorbitan Monolaurate Solution, Wako Pure Chemical Industries, 161-24801 
$1 \mathrm{M}$ Tris- $\mathrm{HCl}(\mathrm{pH} 7.0)$,

Industries, 311-90411

$1 \mathrm{M}$ Tris- $\mathrm{HCl}(\mathrm{pH} 7.5)$,

Industries, 316-90221

$1 \mathrm{M}$ Tris- $\mathrm{HCl}(\mathrm{pH} 8.5)$,

Industries, 316-90405

3M Sodium Acetate ( $\mathrm{pH} 5.2$ ),

Industries, 316-90081

Dimethyl Sulfoxide,

Industries, 046-21981

Ethanol (99.5),

Industries, 057-00456

$2 \mathrm{M} \mathrm{NaOH}$,

Industries, 194-05631

Hybridization buffer HT1,

<Equipment>

Product name,

Cat. No.

Axygen $0.2 \mathrm{~mL}$ Polypropylene PCR Tube Strips PCR-0208-CP-C

and Domed Cap Strips, 8 Tubes/Strip, 8 Domed Caps/Strip,

Clear, Nonsterile

Axygen $1.5 \mathrm{~mL}$ Maxymum Recovery Snaplock

MCT-150-L-C

Microcentrifuge Tube, Polypropylene, Clear, Nonsterile,

250 Tubes/Pack, 10 Packs/Case

Axygen 16 Well Polypropylene PCR Microplate,

PCR-16-C

Clear, Nonsterile

Axygen PCR 1 x 8 Strip Domed Caps,

PCR-02CP-C

Fit $0.2 \mathrm{~mL}$ PCR Tube Strips, Clear, Nonsterile,

$X$-Pierce Sealing Films, Sterile,

XPS-25

$10 \mu \mathrm{L}(0.1-10 \mu \mathrm{L})$ LongReach Barrier tip, 38000T

$10 \mu \mathrm{L}(0.1$ - $10 \mu \mathrm{L})$ Ultra G Barrier tip,

$28200 \mathrm{~T}$

$1000 \mu \mathrm{L}(100-1000 \mu \mathrm{l})$ Barrier tip,

$14200 \mathrm{~T}$

$20 \mu \mathrm{L}(1-20 \mu \mathrm{L})$ Barrier tip,

$14210 \mathrm{~T}$

$200 \mu \mathrm{L}(1-200 \mu \mathrm{L}) \mathrm{NX}$ Barrier tip,
Wako Pure Chemical

Wako Pure Chemical

Wako Pure Chemical

Wako Pure Chemical

Wako Pure Chemical

Wako Pure Chemical

Wako Pure Chemical

Illumina

\section{Maker}

CORNING, AXYGEN

CORNING, AXYGEN

CORNING, AXYGEN

CORNING, AXYGEN

EXCEL Scientific, Inc.

Sorenson BioScience, Inc.

Sorenson BioScience, Inc.

Sorenson BioScience, Inc.

Sorenson BioScience, Inc.

Sorenson BioScience, Inc. 
30550T

MicroAmp Optical 384-Well Reaction Plate with Barcode, ThermoFisher SCIENTIFIC 4309849

MicroAmp Optical Adhesive Film,

ThermoFisher SCIENTIFIC

4360954

PIPETMAN Classic, P1000,

GILSON

F123602

PIPETMAN Classic, P2,

GILSON

F144801

PIPETMAN Classic, P20,

GILSON

F123600

PIPETMAN Classic, P200,

GILSON

F123601

MJ Research Tetrad PTC-225 Thermal Cycler,

GMI, MJ Research

8252-30-1004

MINI CENTRIFUGE,

HARMONY (LMS)

CFM-1000

PlateSpin II,

KUBOTA

Proline Plus Mechanical Pipettes, 8-ch, 0.5 - $10 \mu \mathrm{L}$, 728120

Proline Plus Mechanical Pipettes, 8-ch, 10 - $100 \mu \mathrm{L}$, 728130

Proline Plus Mechanical Pipettes, 8-ch, 30 - $300 \mu \mathrm{L}$, 728140

Vortex-Genie 2,

Scientific Industries, Inc.

SI-0286

miVAC DNA,

SP Scientific, Genevac

DNA-10000-G00

miVac rotor for micro plate,

SP Scientific, Genevac

DRS-00000-200

Dynabeads MPC-S (Magnetic Particle Concentrator), SCIENTIFIC A13346

DynaMag-96 Side Skirted Magnet,

sartorius

sartorius

sartorius

SCIENTIFIC 12027

ThermoFisher

ThermoFisher

$<$ RT primer (for SSCAGE) >

name 5' modification Sequence

N6+TCT P

TCTNNNNNN

3' modification base

9

<RT primers containing barcode (for LQ-sSCAGE)>

name

5 ' modification Sequence

Base 
TCT_7nt_N15_\#001 P

25

TCT_7nt_N15_\#002 P

25

TCT_7nt_N15_\#003 P

25

TCT_7nt_N15_\#004 P

25

TCT_7nt_N15_\#005 P 25

TCT_7nt_N15_\#006 P 25

TCT_7nt_N15_\#007 P

25

TCT_7nt_N15_\#008 P

25

TCT_7nt_N15_\#009 P

25

TCT_7nt_N15_\#010 P

25

TCT_7nt_N15_\#011 P

25

TCT_7nt_N15_\#012 P

25

TCT_7nt_N15_\#013 P

25

TCT_7nt_N15_\#014 P

25

TCT_7nt_N15_\#015 P

25

TCT_7nt_N15_\#016 P

25

TCT_7nt_N15_\#017 P

25

TCT_7nt_N15_\#018 P

25

TCT_7nt_N15_\#019 P 25

TCT_7nt_N15_\#020 P 25

TCT_7nt_N15_\#021 P

25

TCT_7nt_N15_\#022 P

25
TCTGATGCTCNNNNNNNNNNNNNNN

TCTTATGAGCNNNNNNNNNNNNNNN

TCTCGACGATNNNNNNNNNNNNNNN

TCTTAGTCACNNNNNNNNNNNNNNN

TCTCGTACTGNNNNNNNNNNNNNNN

TCTTACGTAGNNNNNNNNNNNNNNN

TCTAGACTCGNNNNNNNNNNNNNNN

TCTTCGCTGANNNNNNNNNNNNNNN

TCTCGATCTGNNNNNNNNNNNNNNN

TCTTGTCACGNNNNNNNNNNNNNNN

TCTATGCACTNNNNNNNNNNNNNNN

TCTGACATCGNNNNNNNNNNNNNNN

TCTATCTAGCNNNNNNNNNNNNNNN

TCTTGTCGCANNNNNNNNNNNNNNN

TCTCAGATCTNNNNNNNNNNNNNNN

TCTTCAGAGCNNNNNNNNNNNNNNN

TCTATACTGCNNNNNNNNNNNNNNN

TCTATCGTGANNNNNNNNNNNNNNN

TCTGAGCTCTNNNNNNNNNNNNNNN

TCTTCTCGAGNNNNNNNNNNNNNNN

TCTCAGTGTCNNNNNNNNNNNNNNN

TCTGCTACGANNNNNNNNNNNNNNN 
TCT_7nt_N15_\#023 P

25

TCT_7nt_N15_\#024 P

25

TCT_7nt_N15_\#025 P

25

TCT_7nt_N15_\#026 P

25

TCT_7nt_N15_\#027 P 25

TCT_7nt_N15_\#028 P 25

TCT_7nt_N15_\#029 P 25

TCT_7nt_N15_\#030 P 25

TCT_7nt_N15_\#031 P 25

TCT_7nt_N15_\#032 P 25

TCT_7nt_N15_\#033 P 25

TCT_7nt_N15_\#034 P 25

TCT_7nt_N15_\#035 P 25

TCT_7nt_N15_\#036 P 25

TCT_7nt_N15_\#037 P

25

TCT_7nt_N15_\#038 P

25

TCT_7nt_N15_\#039 P

25

TCT_7nt_N15_\#040 P 25

TCT_7nt_N15_\#041 P 25

TCT_7nt_N15_\#042 P 25

TCT_7nt_N15_\#043 P 25

TCT_7nt_N15_\#044 P 25
TCTAGTACGCNNNNNNNNNNNNNNN

TCTGACTAGCNNNNNNNNNNNNNNN

TCTTACGCTANNNNNNNNNNNNNNN

TCTGCTATAGNNNNNNNNNNNNNNN

TCTACACTGTNNNNNNNNNNNNNNN

TCTACATGTCNNNNNNNNNNNNNNN

TCTGCAGACTNNNNNNNNNNNNNNN

TCTTAGCGACNNNNNNNNNNNNNNN

TCTACAGTCGNNNNNNNNNNNNNNN

TCTGTATGACNNNNNNNNNNNNNNN

TCTCAGCAGTNNNNNNNNNNNNNNN

TCTACAGCTGNNNNNNNNNNNNNNN

TCTGCTGATANNNNNNNNNNNNNNN

TCTTGTCGACNNNNNNNNNNNNNNN

TCTTGCGATANNNNNNNNNNNNNNN

TCTAGTGTCANNNNNNNNNNNNNNN

TCTATACGTCNNNNNNNNNNNNNNN

TCTCACTATGNNNNNNNNNNNNNNN

TCTTCACAGTNNNNNNNNNNNNNNN

TCTTACGCGTNNNNNNNNNNNNNNN

TCTCGACGTANNNNNNNNNNNNNNN

TCTAGCATGCNNNNNNNNNNNNNNN 
TCT_7nt_N15_\#045 P

25

TCT_7nt_N15_\#046 P

25

TCT_7nt_N15_\#047 P

25

TCT_7nt_N15_\#048 P

25

TCT_7nt_N15_\#049 P 25

TCT_7nt_N15_\#050 P 25

TCT_7nt_N15_\#051 P 25

TCT_7nt_N15_\#052 P 25

TCT_7nt_N15_\#053 P 25

TCT_7nt_N15_\#054 P 25

TCT_7nt_N15_\#055 P 25

TCT_7nt_N15_\#056 P 25

TCT_7nt_N15_\#057 P 25

TCT_7nt_N15_\#058 P 25

TCT_7nt_N15_\#059 P 25

TCT_7nt_N15_\#060 P

25

TCT_7nt_N15_\#061 P

25

TCT_7nt_N15_\#062 P 25

TCT_7nt_N15_\#063 P 25

TCT_7nt_N15_\#064 P 25

TCT_7nt_N15_\#065 P 25

TCT_7nt_N15_\#066 P 25
TCTATCGCGTNNNNNNNNNNNNNNN

TCTGATATGCNNNNNNNNNNNNNNN

TCTTAGCATCNNNNNNNNNNNNNNN

TCTGCTCATGNNNNNNNNNNNNNNN

TCTCTGATGCNNNNNNNNNNNNNNN

TCTGCATATGNNNNNNNNNNNNNNN

TCTGTCACATNNNNNNNNNNNNNNN

TCTCTAGCATNNNNNNNNNNNNNNN

TCTCACGTGTNNNNNNNNNNNNNNN

TCTCTAGTACNNNNNNNNNNNNNNN

TCTGATCGTANNNNNNNNNNNNNNN

TCTCTAGTCGNNNNNNNNNNNNNNN

TCTGACACTGNNNNNNNNNNNNNNN

TCTAGCTCGANNNNNNNNNNNNNNN

TCTGACTGCANNNNNNNNNNNNNNN

TCTAGACGCTNNNNNNNNNNNNNNN

TCTCAGTCTGNNNNNNNNNNNNNNN

TCTTGACTGCNNNNNNNNNNNNNNN

TCTATCAGCGNNNNNNNNNNNNNNN

TCTCTCAGAGNNNNNNNNNNNNNNN

TCTTATGCACNNNNNNNNNNNNNNN

TCTTGCTACANNNNNNNNNNNNNNN 
TCT_7nt_N15_\#067 P

25

TCT_7nt_N15_\#068 P

25

TCT_7nt_N15_\#069 P

25

TCT_7nt_N15_\#070 P

25

TCT_7nt_N15_\#071 P 25

TCT_7nt_N15_\#072 P 25

TCT_7nt_N15_\#073 P 25

TCT_7nt_N15_\#074 P 25

TCT_7nt_N15_\#075 P 25

TCT_7nt_N15_\#076 P 25

TCT_7nt_N15_\#077 P 25

TCT_7nt_N15_\#078 P 25

TCT_7nt_N15_\#079 P 25

TCT_7nt_N15_\#080 P 25

TCT_7nt_N15_\#081 P 25

TCT_7nt_N15_\#082 P

25

TCT_7nt_N15_\#083 P

25

TCT_7nt_N15_\#084 P 25

TCT_7nt_N15_\#085 P 25

TCT_7nt_N15_\#086 P 25

TCT_7nt_N15_\#087 P 25

TCT_7nt_N15_\#088 P 25
TCTATGCGCANNNNNNNNNNNNNNN

TCTTCGTGACNNNNNNNNNNNNNNN

TCTCGATACGNNNNNNNNNNNNNNN

TCTGCTAGCANNNNNNNNNNNNNNN

TCTTAGCGTANNNNNNNNNNNNNNN

TCTCTGACGANNNNNNNNNNNNNNN

TCTTACGTCANNNNNNNNNNNNNNN

TCTCATAGCTNNNNNNNNNNNNNNN

TCTTCGATCANNNNNNNNNNNNNNN

TCTCATATCGNNNNNNNNNNNNNNN

TCTCTCGATANNNNNNNNNNNNNNN

TCTAGTCAGTNNNNNNNNNNNNNNN

TCTTCGATAGNNNNNNNNNNNNNNN

TCTGCGTATANNNNNNNNNNNNNNN

TCTATGTCAGNNNNNNNNNNNNNNN

TCTACGTCGANNNNNNNNNNNNNNN

TCTCACTAGTNNNNNNNNNNNNNNN

TCTTCACGTGNNNNNNNNNNNNNNN

TCTGCTGTACNNNNNNNNNNNNNNN

TCTTACTGAGNNNNNNNNNNNNNNN

TCTCGATGCANNNNNNNNNNNNNNN

TCTTGTCAGANNNNNNNNNNNNNNN 
TCT_7nt_N15_\#089 P

25

TCT_7nt_N15_\#090 P

25

TCT_7nt_N15_\#091 P

25

TCT_7nt_N15_\#092 P

25

TCT_7nt_N15_\#093 P 25

TCT_7nt_N15_\#094 P 25

TCT_7nt_N15_\#095 P 25

TCT_7nt_N15_\#096 P 25

TCT_7nt_N15_\#097 P 25

TCT_7nt_N15_\#098 P 25

TCT_7nt_N15_\#099 P 25

TCT_7nt_N15_\#100 P 25

TCT_7nt_N15_\#101 P 25

TCT_7nt_N15_\#102 P 25

TCT_7nt_N15_\#103 P 25

TCT_7nt_N15_\#104 P

25

TCT_7nt_N15_\#105 P

25

TCT_7nt_N15_\#106 P 25

TCT_7nt_N15_\#107 P 25

TCT_7nt_N15_\#108 P 25

TCT_7nt_N15_\#109 P 25

TCT_7nt_N15_\#110 P 25
TCTCTCTAGANNNNNNNNNNNNNNN

TCTCGTACATNNNNNNNNNNNNNNN

TCTCTAGCTGNNNNNNNNNNNNNNN

TCTCGCTATANNNNNNNNNNNNNNN

TCTTAGCTCANNNNNNNNNNNNNNN

TCTGTATAGCNNNNNNNNNNNNNNN

TCTGTGACTCNNNNNNNNNNNNNNN

TCTAGCATCGNNNNNNNNNNNNNNN

TCTTGAGCATNNNNNNNNNNNNNNN

TCTCGTATAGNNNNNNNNNNNNNNN

TCTACTAGCGNNNNNNNNNNNNNNN

TCTACTGATGNNNNNNNNNNNNNNN

TCTGTACATGNNNNNNNNNNNNNNN

TCTTGACTCANNNNNNNNNNNNNNN

TCTGATCTCANNNNNNNNNNNNNNN

TCTCGACTGTNNNNNNNNNNNNNNN

TCTCAGTAGCNNNNNNNNNNNNNNN

TCTACTATGCNNNNNNNNNNNNNNN

TCTACGATCGNNNNNNNNNNNNNNN

TCTGATCACGNNNNNNNNNNNNNNN

TCTTGCATAGNNNNNNNNNNNNNNN

TCTCGTCATANNNNNNNNNNNNNNN 


$\begin{array}{ll}\text { TCT_7nt_N15_\#111 P } & \text { TCTAGCTCAGNNNNNNNNNNNNNNN } \\ 25 & \\ \text { TCT_7nt_N15_\#112 P } & \text { TCTGTAGCACNNNNNNNNNNNNNNN } \\ 25 & \\ \text { TCT_7nt_N15_\#113 P } & \text { TCTCTATGCGNNNNNNNNNNNNNNN } \\ 25 & \\ \text { TCT_7nt_N15_\#114P } & \text { TCTGACTACTNNNNNNNNNNNNNNN } \\ 25 & \\ \text { TCT_7nt_N15_\#115P } & \text { TCTGATCGCTNNNNNNNNNNNNNNN } \\ 25 & \\ \text { TCT_7nt_N15_\#116P } & \text { TCTCTGATAGNNNNNNNNNNNNNNN } \\ 25 & \\ \text { TCT_7nt_N15_\#117P } & \text { TCTGTGCATANNNNNNNNNNNNNNN } \\ 25 & \\ \text { TCT_7nt_N15_\#118P } & \\ 25 & \text { TCTGATGCATNNNNNNNNNNNNNNN } \\ \text { TCT_7nt_N15_\#119P } & \\ 25 & \text { TCTACTGCAGNNNNNNNNNNNNNNN } \\ \text { TCT_7nt_N15_\#120 P } & \\ 25 & \text { TCTTGATCACNNNNNNNNNNNNNNN }\end{array}$

$<5^{\prime}$ linkers $>$

name, 5' modification, sequence, 3 ' modification, base

5'adaptor N6, -,

AATGATACGGCGACCACCGAGATCTACACTCTTTCCCTACACGACGCTCTTCCGATC TNNNNNN, P, 64

5'adaptor GN5, -,

AATGATACGGCGACCACCGAGATCTACACTCTTTCCCTACACGACGCTCTTCCGATC TGNNNNN, $\mathrm{P}, 64$

5'adaptor down, $P$, AGATCGGAAGAGCGTCGTGTAGGGAAAGAGTGTAGATCTCGGTGGTCGCCGTATCAT $\mathrm{T}, \mathrm{P}, 58$

$<3$ ' linkers >

name

sequence, 3 ' modification, base

3'end adaptor up 01

NNNAGATCGGAAGAGCACACGTCTGAACTCCAGTCACATCACGATCTCGTATGCCG TCTTCTGCTTG, P, 67

3'end adaptor down 01

CAAGCAGAAGACGGCATACGAGATCGTGATGTGACTGGAGTTCAGACGTGTGCTCTT CCGA, - , 61

3'end adaptor up 02 
NNNAGATCGGAAGAGCACACGTCTGAACTCCAGTCACCGATGTATCTCGTATGCCG TCTTCTGCTTG, P, 67

3'end adaptor down 02

CAAGCAGAAGACGGCATACGAGATACATCGGTGACTGGAGTTCAGACGTGTGCTCTT CCGA, - , 61

3'end adaptor up 03

NNNAGATCGGAAGAGCACACGTCTGAACTCCAGTCACTTAGGCATCTCGTATGCCG TCTTCTGCTTG, P, 67

3'end adaptor down 03

CAAGCAGAAGACGGCATACGAGATGCCTAAGTGACTGGAGTTCAGACGTGTGCTCTT CCGA, - , 61

3'end adaptor up 04

NNNAGATCGGAAGAGCACACGTCTGAACTCCAGTCACTGACCAATCTCGTATGCCG TCTTCTGCTTG, P, 67

3'end adaptor down 04

CAAGCAGAAGACGGCATACGAGATTGGTCAGTGACTGGAGTTCAGACGTGTGCTCTT CCGA, - , 61

3'end adaptor up 05

NNNAGATCGGAAGAGCACACGTCTGAACTCCAGTCACACAGTGATCTCGTATGCCG TCTTCTGCTTG, P, 67

3'end adaptor down 05

CAAGCAGAAGACGGCATACGAGATCACTGTGTGACTGGAGTTCAGACGTGTGCTCTT CCGA, - , 61

3'end adaptor up 06

NNNAGATCGGAAGAGCACACGTCTGAACTCCAGTCACGCCAATATCTCGTATGCCG TCTTCTGCTTG, P, 67

3'end adaptor down 06

CAAGCAGAAGACGGCATACGAGATATTGGCGTGACTGGAGTTCAGACGTGTGCTCTT CCGA, - , 61

3'end adaptor up 07

NNNAGATCGGAAGAGCACACGTCTGAACTCCAGTCACCAGATCATCTCGTATGCCG TCTTCTGCTTG, P, 67

3'end adaptor down 07

CAAGCAGAAGACGGCATACGAGATGATCTGGTGACTGGAGTTCAGACGTGTGCTCTT CCGA, - , 61

3'end adaptor up 08

NNNAGATCGGAAGAGCACACGTCTGAACTCCAGTCACACTTGAATCTCGTATGCCG TCTTCTGCTTG, P, 67

3'end adaptor down 08

CAAGCAGAAGACGGCATACGAGATTCAAGTGTGACTGGAGTTCAGACGTGTGCTCTT CCGA, - , 61

3'end adaptor up 09

NNNAGATCGGAAGAGCACACGTCTGAACTCCAGTCACGATCAGATCTCGTATGCCG TCTTCTGCTTG, P, 67 
3'end adaptor down 09

CAAGCAGAAGACGGCATACGAGATCTGATCGTGACTGGAGTTCAGACGTGTGCTCTT CCGA, - , 61

3'end adaptor up 10

NNNAGATCGGAAGAGCACACGTCTGAACTCCAGTCACTAGCTTATCTCGTATGCCG TCTTCTGCTTG, P, 67

3'end adaptor down 10

CAAGCAGAAGACGGCATACGAGATAAGCTAGTGACTGGAGTTCAGACGTGTGCTCTT CCGA, - , 61

3'end adaptor up 11

NNNAGATCGGAAGAGCACACGTCTGAACTCCAGTCACGGCTACATCTCGTATGCCG TCTTCTGCTTG, P, 67

3'end adaptor down 11

CAAGCAGAAGACGGCATACGAGATGTAGCCGTGACTGGAGTTCAGACGTGTGCTCTT CCGA, - , 61

3'end adaptor up 12

NNNAGATCGGAAGAGCACACGTCTGAACTCCAGTCACCTTGTAATCTCGTATGCCG TCTTCTGCTTG, P, 67

3'end adaptor down 12

CAAGCAGAAGACGGCATACGAGATTACAAGGTGACTGGAGTTCAGACGTGTGCTCTT CCGA, - , 61

3'end adaptor up 13

NNNAGATCGGAAGAGCACACGTCTGAACTCCAGTCACAGTCAACAATCTCGTATGC CGTCTTCTGCTTG, P, 69

3'end adaptor down 13

CAAGCAGAAGACGGCATACGAGATTGTTGACTGTGACTGGAGTTCAGACGTGTGCTC TTCCGA, - , 63

3'end adaptor up 14

NNNAGATCGGAAGAGCACACGTCTGAACTCCAGTCACAGTTCCGTATCTCGTATGC CGTCTTCTGCTTG, P, 69

3'end adaptor down 14

CAAGCAGAAGACGGCATACGAGATACGGAACTGTGACTGGAGTTCAGACGTGTGCT CTTCCGA, - , 63

3'end adaptor up 15

NNNAGATCGGAAGAGCACACGTCTGAACTCCAGTCACATGTCAGAATCTCGTATGC CGTCTTCTGCTTG, P, 69

3'end adaptor down 15

CAAGCAGAAGACGGCATACGAGATTCTGACATGTGACTGGAGTTCAGACGTGTGCTC TTCCGA, - , 63

3'end adaptor up 16

NNNAGATCGGAAGAGCACACGTCTGAACTCCAGTCACCCGTCCCGATCTCGTATGC CGTCTTCTGCTTG, P, 69

3'end adaptor down 16

CAAGCAGAAGACGGCATACGAGATCGGGACGGGTGACTGGAGTTCAGACGTGTGCT 
CTTCCGA, - , 63

3'end adaptor up 18

NNNAGATCGGAAGAGCACACGTCTGAACTCCAGTCACGTCCGCACATCTCGTATGC CGTCTTCTGCTTG, P, 69

3'end adaptor down 18

CAAGCAGAAGACGGCATACGAGATGTGCGGACGTGACTGGAGTTCAGACGTGTGCT

CTTCCGA, - , 63

3'end adaptor up 19

NNNAGATCGGAAGAGCACACGTCTGAACTCCAGTCACGTGAAACGATCTCGTATGC CGTCTTCTGCTTG, P, 69

3'end adaptor down 19

CAAGCAGAAGACGGCATACGAGATCGTTTCACGTGACTGGAGTTCAGACGTGTGCTC TTCCGA, - , 63

3'end adaptor up 20

NNNAGATCGGAAGAGCACACGTCTGAACTCCAGTCACGTGGCCTTATCTCGTATGC CGTCTTCTGCTTG, P, 69

3'end adaptor down 20

CAAGCAGAAGACGGCATACGAGATAAGGCCACGTGACTGGAGTTCAGACGTGTGCT

CTTCCGA, - , 63

3 'end adaptor up 21

NNNAGATCGGAAGAGCACACGTCTGAACTCCAGTCACGTTTCGGAATCTCGTATGC

CGTCTTCTGCTTG, P, 69

3'end adaptor down 21

CAAGCAGAAGACGGCATACGAGATTCCGAAACGTGACTGGAGTTCAGACGTGTGCT

CTTCCGA, - , 63

3'end adaptor up 22

NNNAGATCGGAAGAGCACACGTCTGAACTCCAGTCACCGTACGTAATCTCGTATGC CGTCTTCTGCTTG, P, 69

3'end adaptor down 22

CAAGCAGAAGACGGCATACGAGATTACGTACGGTGACTGGAGTTCAGACGTGTGCTC TTCCGA, - , 63

3'end adaptor up 23

NNNAGATCGGAAGAGCACACGTCTGAACTCCAGTCACGAGTGGATATCTCGTATGC CGTCTTCTGCTTG, P, 69

3'end adaptor down 23

CAAGCAGAAGACGGCATACGAGATATCCACTCGTGACTGGAGTTCAGACGTGTGCTC TTCCGA, - , 63

3'end adaptor up 25

NNNAGATCGGAAGAGCACACGTCTGAACTCCAGTCACACTGATATATCTCGTATGC CGTCTTCTGCTTG, P, 69

3'end adaptor down 25

CAAGCAGAAGACGGCATACGAGATATATCAGTGTGACTGGAGTTCAGACGTGTGCTC TTCCGA, - , 63

3 'end adaptor up 27 
NNNAGATCGGAAGAGCACACGTCTGAACTCCAGTCACATTCCTTTATCTCGTATGCC GTCTTCTGCTTG, P, 69

3'end adaptor down 27

CAAGCAGAAGACGGCATACGAGATAAAGGAATGTGACTGGAGTTCAGACGTGTGCT

CTTCCGA, - , 63

\section{<Recipes>}

\section{1. $250 \mathrm{mM} \mathrm{NaIO}_{4}$}

Dissolve $1 \mathrm{mg}$ of Sodium periodate (ACS Reagent Grade) in $18.7 \mu \mathrm{L}$ of RNase/DNase free water and keep in the dark. NOTE: The solution can be aliquoted to $50 \mu \mathrm{L}$ and stored at $80^{\circ} \mathrm{C}$.

\section{2. $\mathbf{1 0 0} \mathrm{mM}$ Biotin (long arm) Hydrazide}

Dissolve $50 \mathrm{mg}$ of Biotin (long arm) Hydrazide in $1.345 \mathrm{~mL}$ of DMSO $1.345 \mathrm{ml}$.

Biotin is packed in $50 \mathrm{mg} /$ tube.

NOTE: The solution can be aliquoted to $50 \mu \mathrm{L}$ and stored at $80^{\circ} \mathrm{C}$.

\section{LiCl buffer}

Mix following materials and store at room temperature.

$\begin{array}{lc}\text { 8M Lithium chloride solution } & 3.64 \mathrm{~mL} \\ 1 \mathrm{M} \text { Tris- } \mathrm{HCl}(\mathrm{pH} 7.5) & 0.80 \mathrm{~mL} \\ \text { 10w/v\% Polyoxyethylene (20) Sorbitan Monolaurate Solution } & 0.40 \mathrm{~mL} \\ \text { 0.5 M EDTA (pH 8.0) } & 0.16 \mathrm{~mL} \\ \text { RNase, DNase - free water } & 3.64 \mathrm{~mL}\end{array}$

\section{TE wash buffer}

Mix following materials and store at room temperature.

RNase, DNase -free water

$9.12 \mathrm{~mL}$

$1 \mathrm{M}$ Tris-HCl ( $\mathrm{pH} 7.5)$

$0.40 \mathrm{~mL}$

$10 \mathrm{w} / \mathrm{v} \%$ Polyoxyethylene (20) Sorbitan Monolaurate Solution $0.40 \mathrm{~mL}$

$0.5 \mathrm{M}$ EDTA ( $\mathrm{pH} 8.0) \quad 0.08 \mathrm{~mL}$

\section{Release buffer}

Mix following materials and store at room temperature.

RNase ONE 10x Reaction Buffer

$100 \mu \mathrm{L}$

$10 \mathrm{w} / \mathrm{v} \%$ Polyoxyethylene (20) Sorbitan Monolaurate Solution $1.0 \mu \mathrm{L}$

RNase, DNase -free water

$899 \mu \mathrm{L}$

\section{6. $1 \times$ TE buffer}

Mix following materials and store at room temperature. 
$1 \mathrm{M}$ Tris- $\mathrm{HCl}(\mathrm{pH} 8.0) \quad 500 \mu \mathrm{L}$

$0.5 \mathrm{M} \mathrm{EDTA}(\mathrm{pH} \mathrm{8.0)} \quad 100 \mu \mathrm{L}$

RNase, DNase-free water $49.4 \mathrm{~mL}$

\section{0.1 M NaCl/TE buffer}

$1 \mathrm{M} \mathrm{NaCl} \quad 500 \mu \mathrm{L}$

$1 \times$ TE buffer $\quad 4.5 \mathrm{~mL}$

\section{8. $2.5 \mu \mathrm{M}$ 5'linker}

a. Mix following solution and carry out the annealing reaction to generate $100 \mu \mathrm{M}$

GN5 linker

$1 \mathrm{mM}$ 5'adaptor GN5 $4 \mu \mathrm{L}$

$1 \mathrm{mM}$ 5'adaptor down $4 \mu \mathrm{L}$

$1 \mathrm{M} \mathrm{NaCl} \quad 4 \mu \mathrm{L}$

$1 \times$ TE buffer $\quad 28 \mu \mathrm{L}$

$<$ Annealing condition>

$95^{\circ} \mathrm{C}, 5 \mathrm{~min}:-0.1^{\circ} \mathrm{C} \mathrm{sec}-1$ down to $83^{\circ} \mathrm{C} ; 5 \mathrm{~min}$ at $83^{\circ} \mathrm{C}$; $-0.1{ }^{\circ} \mathrm{C}$ sec- 1 down to 71

${ }^{\circ} \mathrm{C}$; 5 min at $71{ }^{\circ} \mathrm{C}$; $-0.1{ }^{\circ} \mathrm{C}$ sec- 1 down to $59^{\circ} \mathrm{C}$; 5 min at $59{ }^{\circ} \mathrm{C}$; $-0.1{ }^{\circ} \mathrm{C}$ sec- 1 , to 47

${ }^{\circ} \mathrm{C}$; $5 \mathrm{~min}$ at $47^{\circ} \mathrm{C} ;-0.1{ }^{\circ} \mathrm{C} \mathrm{sec}-1$, to $35^{\circ} \mathrm{C} ; 5 \mathrm{~min}$ at $35^{\circ} \mathrm{C} ;-0.1^{\circ} \mathrm{C} \mathrm{s}-1$ to $23{ }^{\circ} \mathrm{C} ; 5 \mathrm{~min}$ at $23^{\circ} \mathrm{C} ;-0.1^{\circ} \mathrm{C} \mathrm{sec}-1$ to $11^{\circ} \mathrm{C}$, and then hold at $11^{\circ} \mathrm{C}$.

b. Mix following solutions and repeat the annealing reaction at step a. to generate $100 \mu \mathrm{M}$ N6 linker

$1 \mathrm{mM}$ 5'adaptor N6 $1 \mu \mathrm{L}$

$1 \mathrm{mM} 5$ 'adaptor down $1 \mu \mathrm{L}$

$1 \mathrm{M} \mathrm{NaCl} \quad 1 \mu \mathrm{L}$

$1 \times$ TE buffer $\quad 7 \mu \mathrm{L}$

c. Mix $40 \mu \mathrm{L}$ of $100 \mu \mathrm{M}$ GN5 linker from step a. and $10 \mu \mathrm{L}$ of $100 \mu \mathrm{M}$ N6 linker from step b.

d. Dilute $100 \mu \mathrm{M}$ of mixed 5'linkers to $2.5 \mu \mathrm{M}$ in $0.1 \mathrm{M} \mathrm{NaCl} / \mathrm{TE}$ buffer. For instance, add $2.5 \mu \mathrm{L}$ of $100 \mu \mathrm{M}$ mixed linkers from step c to $97.5 \mu \mathrm{L}$ of $0.1 \mathrm{M} \mathrm{NaCl} / \mathrm{TE}$ buffer for 25 samples.

NOTE: $2.5 \mu \mathrm{M}$ and $100 \mathrm{M}$ mixed $5^{\prime}$ linkers can be stored at $-20^{\circ} \mathrm{C}$.

9. $2.5 \mu \mathrm{M} 3^{\prime}$ linker

a. Mix following solutions and carry out the annealing reaction at the step 8.a. to generate $100 \mu \mathrm{M}$ of $3^{\prime}$ linker.

$1 \mathrm{mM}$ 3'end adaptor up $1 \mu \mathrm{L}$ 
$1 \mathrm{mM}$ 3'end adaptor down $1 \mu \mathrm{L}$

$1 \mathrm{M} \mathrm{NaCl} \quad 1 \mu \mathrm{L}$

$1 \times$ TE buffer $\quad 7 \mu \mathrm{L}$

b. Dilute $100 \mu \mathrm{M}$ mixed linker from step a. to $2.5 \mu \mathrm{M}$ in $0.1 \mathrm{M} \mathrm{NaCl} / \mathrm{TE}$ buffer (see details at the step 8.d.).

\section{BEFORE START INSTRUCTIONS}

RNAs should be treated in the RNAse free solutions, benches and equipments. We highly recommend to perform RNA quality check before starting (see detail at previously published paper RNA stability check).

RT reaction in different well using $R T$ primer with barcode

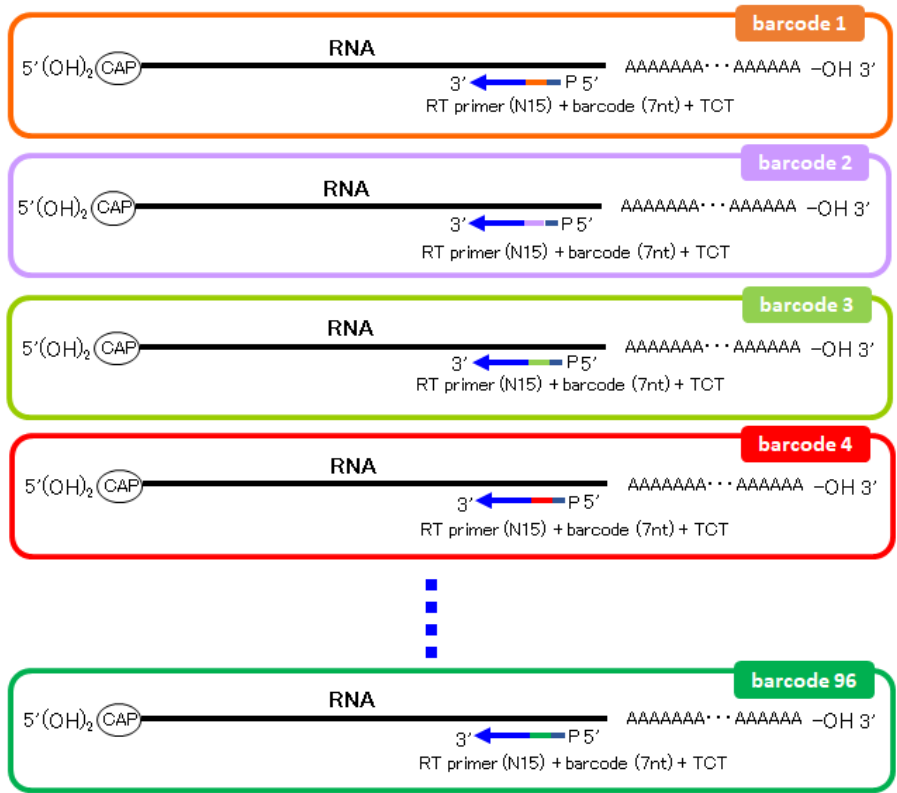

Step 1.2

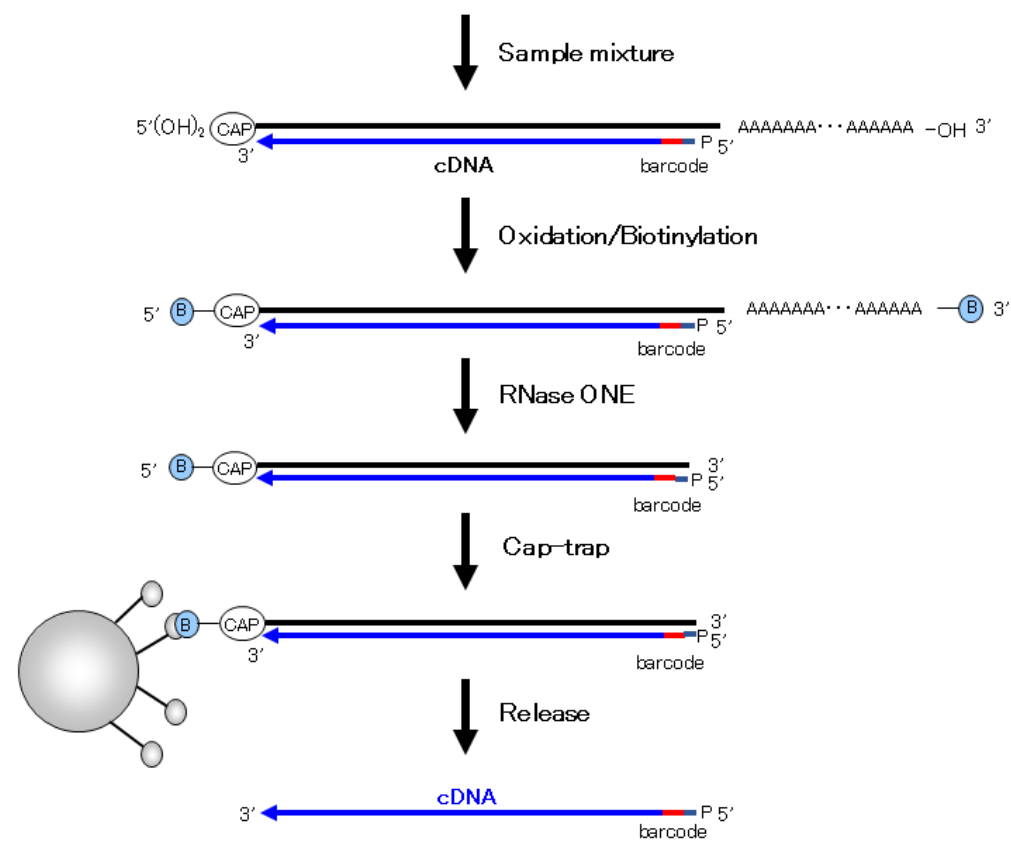

Step 2-4

Step 5

Step 6-8 


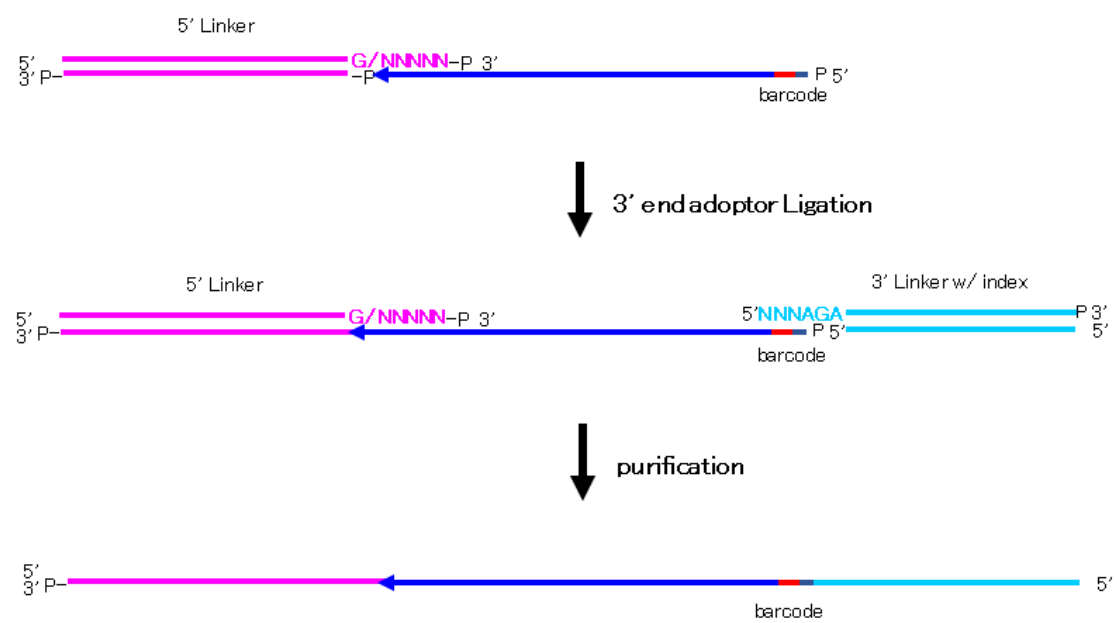

Figure.1 Protocol Flow

\section{Reverse Transcription (RT) to generate RNA/cDNA hybrids}

\section{1 single strand CAGE (ssCAGE)}

II) $\mu \mathrm{g}$ starting RNAs.

When the amount of RNAs is less than $1 \mu \mathrm{g}$, go to step 1.2.

a. Mix $5 \mu \mathrm{L}$ of RNAs $(1 \mu \mathrm{g} / \mu \mathrm{L}), 1 \mu \mathrm{L}$ of RT primer (N6+TCT) and $4 \mu \mathrm{L}$ of RNase free water in a tube.

\section{Note}

NOTE: Use $1.5 \mathrm{~mL}$ tube, 8 strip PCR tube or $16-96$ wells plate depending on numbers of samples.

b. Incubate the RNA/primer mix from step a. at $65^{\circ} \mathrm{C}$ for $5 \mathrm{~min}$ and immediately put on ice for $2 \min$

c. Add the following components to the solution from step $\mathbf{b}$. and carefully mix by pipetting on ice.

$5 \times$ First-Strand buffer

$4 \mu \mathrm{L}$ 
$10 \mathrm{mM}$ dNTPs $\quad 1 \mu \mathrm{L}$

$0.1 \mathrm{M} \mathrm{DTT}$

RNase free water $\quad 2 \mu \mathrm{L}$

SuperScript III Reverse Transcriptase $2 \mu \mathrm{L}$

d. Incubate the mixed solution (total $20 \mu \mathrm{L}$ ) at $25^{\circ} \mathrm{C}$ for $30 \mathrm{sec}, 55^{\circ} \mathrm{C}$ for $30 \mathrm{~min}$ and keep $4{ }^{\circ} \mathrm{C}$ to generate RNA/cDNA hybrids.

e. Add $36 \mu \mathrm{L}$ (1.8-folds) of RNACleanXP beads to $20 \mu \mathrm{L}$ of RNA/cDNA hybrids from step d. and mix well by pipetting.

(1). Incubate at room temperature for $5 \mathrm{~min}$. Spin down and set the tube on magnetic bar for 5 $\min$.

(2). Discard the supernatant by pipette aspiration.

(3). Wash the beads with $200 \mu \mathrm{L}$ of $70 \% \mathrm{EtOH}$.

(4). Set the tube on magnetic bar for $5 \mathrm{~min}$.

(5). Discard the supernatant by pipette aspiration.

(6. Repeat step (3) (5).

7). Discard the $70 \%$ EtOH completely by pipette aspiration.

8. Add $42 \mu \mathrm{L}$ of RNase free water and mix by pipetting extensively (more than 60 times) to elute RNA/cDNA hybrids.

(9. Incubate at room temperature for $5 \mathrm{~min}$.

(10.) Spin down and set the tube on magnetic bar for $5 \mathrm{~min}$.

(11). Transfer supernatant ( $\sim 40 \mathrm{~L}$ of the cDNA/RNA hybrids) to new tube.

1.2 Low Quantity single strand CAGE (LQ-ssCAGE)

When the amount of starting RNAs is less than $1 \mu \mathrm{g}$, choose this protocol.

Note

NOTE: The amount of RNAs in one tube should be $25 \mathrm{ng} \sim 100 \mathrm{ng}$ for the RT reaction.

After RT, samples have each barcode and can be mixed up to $5 \mu \mathrm{g}$. For instance, if the starting RNA amount is $50 \mathrm{ng}, 100$ samples can be mixed. The number of mixed samples is depending on how much samples you have and how deep the samples should be sequenced. Here is an example of 48 mix samples from each $50 \mathrm{ng}$ starting RNA.

CRITICAL: The mixed solution should be less than $5 \mu \mathrm{g}$. Otherwise the following linkers at step 9 and $\mathbf{1 1}$ are not enough for the linker ligation.

a. Mix $4 \mu \mathrm{L}$ of $50 \mathrm{ng}$ RNAs $(12.5 \mathrm{ng} / \mu \mathrm{L})$ and $1 \mu \mathrm{L}$ of $1.25 \mathrm{mM}$ RT primer containing barcode in a 96 wells plate by pipetting.

b. Incubate the RNA/primer mix form step a. at $65^{\circ} \mathrm{C}$ for $5 \mathrm{~min}$ and immediately put on ice for 2 min.

c. Mix the following components (enzyme mix) on ice: 


$\begin{array}{lc}5 \text { x First Strand buffer } & 2.0 \mu \mathrm{L} \\ 0.1 \mathrm{M} \text { DTT } & 0.5 \mu \mathrm{L} \\ 10 \mathrm{mM} \text { dNTPs } & 0.5 \mu \mathrm{L} \\ \text { RNase free water } & 1.0 \mu \mathrm{L} \\ \text { SuperScript III Reverse Transcriptase } 1.0 \mu \mathrm{L} \\ \text { Total volume } & 5.0 \mu \mathrm{L}\end{array}$

Note

NOTE: Make premix solution for 48 samples $x 1.1$ samples.

d. Add $5 \mu \mathrm{L}$ of enzyme mix from premix solution at step c. to RNA/primer mix from step $\mathbf{b}$. and carefully mix 10 times by pipetting on ice.

e. Incubate at $25^{\circ} \mathrm{C}$ for $30 \mathrm{sec}, 50^{\circ} \mathrm{C}$ for $30 \mathrm{~min}$ and keep $4{ }^{\circ} \mathrm{C}$ to generate RNA/cDNA hybrids.

f. Mix samples by following steps.

(1). Transfer each $10 \mu \mathrm{L}$ of RNA/cDNA hybrids from step e. to one of new $1.5 \mathrm{~mL}$ tube (total volume is $480 \mu \mathrm{L}$ ).

(2). Add $15 \mu \mathrm{L}$ of RNase free water to the first 8 wells at the 96 wells plate from step (1), wash wells by pipetting, and transfer the $15 \mu \mathrm{L}$ of the solutions to the next 8 wells.

3. Wash the 8 wells by pipetting and transfer $15 \mu \mathrm{L}$ of the solutions to the next 8 wells.

(4). Repeat step (2)three times until the end of 8 wells and transfer all solutions (total volume is $120 \mu \mathrm{L}(15 \mu \mathrm{L} \times 8$ wells)) to the $1.5 \mathrm{~mL}$ tube from step (1)(final volume is $600 \mu \mathrm{L}$ ).

(5). Mix $600 \mu \mathrm{L}$ of the solution from step (4) by vortex, spin down and aliquot $200 \mu \mathrm{L}$ in new two tubes of $1.5 \mathrm{~mL}$ tube (total $200 \mu \mathrm{L}$ in 3 tubes of $1.5 \mathrm{~mL}$ tube).

\section{Note}

NOTE: Step $\mathbf{f}$. is for the collection of remaining molecules to avoid losing any RNA/cDNA hybrids in the wells. We recommend to aliquot less than $200 \mu \mathrm{L}$ per each $1.5 \mathrm{~mL}$ tube at step $\mathbf{f}$. (5)due to the volume limitations of next RNAClean XP purification step.

g. Add $300 \mu \mathrm{L}$ ( 1.8 -folds) of RNAClean XP beads to the 48 mixed RNA/cDNA hybrids in a 1.5 $\mathrm{mL}$ tube from step f. (5), mix well by pipetting and then elute the mixed RNA/cDNA hybrids by following steps.

(1.) Incubate at room temperature for $10 \mathrm{~min}$, spin down and set the $1.5 \mathrm{~mL}$ tube on magnetic bar for $5 \mathrm{~min}$.

(2). Discard the supernatant by pipette aspiration.

(3). Wash the beads with $1.2 \mathrm{~mL}$ of $70 \% \mathrm{EtOH}$.

(4). Set the $1.5 \mathrm{~mL}$ tube on magnetic bar for $5 \mathrm{~min}$.

(5). Discard the supernatant by pipette aspiration.

(6. Repeat step (3) (5) twice. 
(7). Discard the $70 \%$ EtOH completely by pipette aspiration.

8. Add $100 \mu \mathrm{L}$ RNase free water and mix by pipetting extensively (more than 60 times) to elute RNA/cDNA hybrids.

(9. Incubate at room temperature for $5 \mathrm{~min}$.

(10.) Spin down and set the tube on magnetic bar for $5 \mathrm{~min}$.

(11). Transfer $100 \mu \mathrm{L}$ of the cDNA/RNA hybrids to new $1.5 \mathrm{~mL}$ tube.

(12). Repeat step (8) (11) twice (final volume is $200 \mu \mathrm{L}$ ).

(13). Concentrate $200 \mu \mathrm{L}$ of the cDNA/RNA hybrids solutions from step (12) to around $40 \mu \mathrm{L}$ by SpeedVac vacuum concentrator at $37^{\circ} \mathrm{C}$, and collect the solutions from 3 of $1.5 \mathrm{~mL}$ tubes to 1 of $1.5 \mathrm{~mL}$ tube (total volume is around $120 \mu \mathrm{L}$ ) and concentrate to $40 \mu \mathrm{L}$ in the $1.5 \mathrm{~mL}$ tube at $37^{\circ} \mathrm{C}$. The timing is around $2 \mathrm{~h}$.

Note

CRITICAL: DO NOT DRY UP the CDNA/RNA hybrids solutions by the concentrating step (13). Check the sample volume during the concentration several times and adjust the final volume to $40 \mu \mathrm{L}$ with RNase/DNase-free when the volume become less than $40 \mu \mathrm{L}$.

\section{Oxidation to modify diol group of cap structure}

a. Mix $40 \mu \mathrm{L}$ of RNA/cDNA hybrid from step 1.1e. or 1.2g., $2 \mu \mathrm{L}$ of $1 \mathrm{M} \mathrm{NaOAc}(\mathrm{pH} 4.5)$ and $2 \mu \mathrm{L}$ of $250 \mathrm{mM} \mathrm{NaIO}_{4}$ in a tube by 10 times pipetting.

Note

NOTE: $1.5 \mathrm{~mL}$ tube, 8-Strip PCR tube or16 wells plate can be used depending on how many samples should be prepared.

b. Incubate for $5 \mathrm{~min}$ on ice in dark by aluminum foil wrapping.

c. Add $16 \mu \mathrm{L}$ of $1 \mathrm{M}$ Tris- $\mathrm{HCl}(\mathrm{pH}$ 8.5) to neutralize the solution and mix well by pipetting.

\section{Purification}

(1)

Add $108 \mu \mathrm{L}$ (1.8 -folds) of RNACleanXP beads to $60 \mu \mathrm{L}$ of oxidated RNA/cDNA hybrid from step 2, mix well by pipetting and then elute the 48 samples mixed RNA/cDNA hybrids by following steps.

(1). Incubate at room temperature for $5 \mathrm{~min}$.

(2). Spin down and set the tube on magnetic bar for $5 \mathrm{~min}$.

(3). Discard the supernatant by pipette aspiration.

(4). Wash the beads with $200 \mu \mathrm{L}$ of $70 \% \mathrm{EtOH}$

(5). Discard the $70 \% \mathrm{EtOH}$.

๔. Repeat step (4) (5)twice and discarded 70\% EtOH completely.

(7). Add $42 \mu \mathrm{L}$ of RNase free water and mix by pipetting extensively (more than 60 times) to elute 
supernatant.

8. Incubate at room temperature for $5 \mathrm{~min}$.

9. Spin down and set the tube on magnetic bar for $5 \mathrm{~min}$.

(10. Collect $40 \mu \mathrm{L}$ of the supernatant to new tube.

\section{Biotinylation by the coupling reaction to the oxidized RNA/cDNA hybrids}

(II)

a. Mix $40 \mu \mathrm{L}$ of purified oxidized RNA/cDNA hybrid from step $3,4 \mu \mathrm{L}$ of $1 \mathrm{M} \mathrm{NaOAc}(\mathrm{pH} 6.3)$ and 4 $\mu \mathrm{L}$ of $100 \mathrm{mM}$ Biotin (long arm) hydrazide by 10 times pipetting.

b. Incubate for $30 \mathrm{~min}$ at $40^{\circ} \mathrm{C}$.

c. Add $86.4 \mu \mathrm{L}$ of RNACleanXP (1.8 -folds) to $44 \mu \mathrm{L}$ of solution from step $b$, and perform purification by following step 3 (1) (10).

Note

NOTE: Biotinylation happens both 5'end of capped RNAs and 3'end of RNA (see Figure 2 at published protocol).

\section{RNaseONE treatment to digest RNA of RNA/cDNA hybrids}

III Add $4.5 \mu \mathrm{L}$ of $10 \times$ RNaseONE buffer and $0.5 \mu \mathrm{L}$ of RNaseONE to $40 \mu \mathrm{L}$ of purified biotinylated RNA/cDNA hybrids from step 4 and mix 10 times by pipetting.

b. Incubate for $30 \mathrm{~min}$ at $37^{\circ} \mathrm{C}$.

c. Add $81 \mu \mathrm{L}$ of RNACleanXP (1.8 -folds) to the solution from step b. and purify by the following step 3 (1) (10).

\section{Note}

CRITICAL: This step is critical to digest uncompleted cDNA synthesis to the $5^{\prime}$ end of capped RNAs (See Figure 2 at published protocol).

\section{Dynabeads M-270 Streptavidin beads preparation}

a. Add $30 \mu \mathrm{L}$ of Dynabeads M-270 Streptavidin to new $1.5 \mathrm{ml}$ tube, set on the magnetic bar for 5 min, and discard supernatant.

b. Wash the beads with $30 \mu \mathrm{L}$ of LiCl buffer and discard supernatant.

c. Repeat step b. for 2 times.

d. Resuspend the beads in $35 \mu \mathrm{L}$ of LiCl buffer.

\section{$7 \quad$ CapTrap reaction}



a. Add $35 \mu \mathrm{L}$ of beads from step 6 to $40 \mu \mathrm{L}$ of RNA/cDNA hybrids from step 5 and mix well by pipetting.
b. Incubate for 15 minutes at $37^{\circ} \mathrm{C}$ and mix by pipetting at the time of $7 \mathrm{~min}$.
c. Spin down and set the plate on the magnetic bar for $2 \mathrm{~min}$ and discard the supernatant by the pipette aspiration.
d. Add $150 \mu \mathrm{L}$ of TE wash buffer and mix 60 times by pipetting. Spin down and set the plate on the magnetic bar for $2 \mathrm{~min}$ and discard the supernatant.
e. Repeat step d. for 3 times.
f. Add $35 \mu \mathrm{L}$ of Release buffer to the beads and mix 60 times by pipetting.
g. Incubate for $5 \mathrm{~min}$ at $95^{\circ} \mathrm{C}$ for $5 \mathrm{~min}$ and on ice for $1 \mathrm{~min}$.
h. Spin down and set the tube on the magnetic bar for $2 \mathrm{~min}$.
i. Transfer the supernatant containing CapTrapped cDNA from step $\mathbf{h}$. to new 16 well plate.
j. Add $30 \mu \mathrm{L}$ of Release buffer to the beads and mix well by pipetting, spin down, and set the tube on the magnetic stand for $2 \mathrm{~min}$.
k. Transfer $30 \mu \mathrm{L}$ of supernatant containing CapTrapped cDNA to new tube.

\section{RNaseONE and RNaseH reaction to remaining RNAs}

a. Add $2.9 \mu \mathrm{L}$ of release buffer, $2 \mu \mathrm{L}$ of RNaseONE and $0.1 \mu \mathrm{L}$ of RNase $\mathrm{H}$ to $30 \mu \mathrm{L}$ of CapTrapped cDNA from step 7 and mix 10 times by pipetting.

b. Incubate at $37^{\circ} \mathrm{C}$ for $30 \mathrm{~min}$.

c. Add $126 \mu \mathrm{L}$ of AMPureXP (1.8 -folds) to $33 \mu \mathrm{L}$ of cDNA from step $\mathbf{b}$. and perform purification by following step 3 (1) (10).

d. Dry up $40 \mu \mathrm{L}$ of purified CapTrapped cDNA by the SpeedVac concentrator at $37^{\circ} \mathrm{C}$ for around 75 min.

e. Add $4 \mu \mathrm{L}$ of RNase free water to the dried pellet.

\section{$9 \quad 5$ ' Single Strand Linker Ligation}
a. Incubate $4 \mu \mathrm{L}$ of $\mathrm{cDNA}$ at $95^{\circ} \mathrm{C}$ for $5 \mathrm{~min}$ and put on ice for $2 \mathrm{~min}$.
b. Incubate $4 \mu \mathrm{L}$ of $5^{\prime}$ linker at $55^{\circ} \mathrm{C}$ for $5 \mathrm{~min}$ and put on ice for $2 \mathrm{~min}$.
c. Mix $4 \mu \mathrm{L}$ of $5^{\prime}$ linker, $4 \mu \mathrm{L}$ of CapTrapped cDNA from step a. and $16 \mu \mathrm{L}$ of Mighty Mix by pipetting (total volume is $24 \mu \mathrm{L}$ ).
d. Incubate at $16^{\circ} \mathrm{C}$ for $16 \mathrm{~h}$ (overnight).

\section{Purification to remove excess $5^{\prime}$ linkers and linker dimers}

a. Add $46 \mu \mathrm{L}$ of RNase free water and $126 \mu \mathrm{L}$ of AMPureXP (1.8 -folds) to $24 \mu \mathrm{L}$ of cDNA from step $\mathbf{9}$, mix well by pipetting.

b. Purify the solution by following step 3 (1) (10.

c. Incubate at $95^{\circ} \mathrm{C}$ for 5 min and immediately put on ice for 2 min.

d. Add $48 \mu \mathrm{L}$ of AMPureXP beads ( 1.2 -folds) to $40 \mu \mathrm{L}$ of CDNA and mix well by pipetting.

e. Repeat purification by following step 3 (1) (10).

f. Dry up $40 \mu \mathrm{L}$ of $\mathrm{cDNA}$ by SpeedVac concentrator at $80^{\circ} \mathrm{C}$ for around $35 \mathrm{~min}$.

g. Add $4 \mu \mathrm{L}$ of RNase free water to the dried pellet. 
CRITICAL: It is important to perform heat inactivation and repeat purification step to remove non-ligated excess linkers during the purification step. Otherwise, these excess linkers can disturb library sequencing quality; excess linkers compete with the library to anneal Illumina flow-cells. In addition, 5'linker-3'linker dimers can be created at the next step 11.

\section{3' Single Strand Linker Ligation}

a. Incubate $4 \mu \mathrm{L}$ of $\mathrm{cDNA}$ at $95^{\circ} \mathrm{C}$ for $5 \mathrm{~min}$ and put on ice for $2 \mathrm{~min}$.

b. Incubate $4 \mu \mathrm{L}$ of $3^{\prime}$ linker at $65^{\circ} \mathrm{C}$ for $5 \mathrm{~min}$ and put on ice for $2 \mathrm{~min}$.

c. Mix $4 \mu \mathrm{L}$ of $3^{\prime}$ linker, $4 \mu \mathrm{L}$ of cDNA from step a. and $16 \mu \mathrm{L}$ of Mighty Mix by pipetting.

d. Incubate at $30^{\circ} \mathrm{C}$ for $4 \mathrm{~h}$.

Note

NOTE: When you have more than 2 libraries with same barcode at RT primer and plan to mix libraries at the sequencing step 14, use different Index at the $3^{\prime}$ linker.

\section{Purification to remove excess 3 ' linkers and linker dimers (final library)}

II b. Dissolve the pellet with $10 \mu \mathrm{L}$ of RNase, DNase-free water.

\section{Library QC}

Quantify the concentration of library by KAPA Library Quantification Kit.

\section{Sequencing}

a. Mix the following components

$\begin{array}{lc}\text { Library }(2250 \text { amol }) & \mathrm{X} \mu \mathrm{L} \\ \text { RNase, DNase-free water } & (19-\mathrm{X}) \mu \mathrm{L} \\ \text { 2N NaOH } & 1 \mu \mathrm{L} \\ \text { Total } & 20 \mu \mathrm{L}\end{array}$

b. Incubate at room temperature for $5 \mathrm{~min}$.

c. Put the tube on ice and add $20 \mu \mathrm{L}$ of $1 \mathrm{M} \mathrm{Tris-} \mathrm{HCl}$ ( $\mathrm{pH} 7.0$, pre-chilled) to neutralize.

d. Add $110 \mu \mathrm{L}$ of HT1 buffer. 
e. Transfer $120 \mu \mathrm{L}$ of the denatured and diluted library to HiSeq2500.

\section{Sequencing kit}

- sSCAGE: 50 bp Single Read sequencing.

- LQ-ssCAGE: 50 bp Paired-End sequencing (see Figure 1 for the library structure). Read 1: to read sequence information of CDNA

Read 2: to read sequence information of barcode in RT primer Index 1: to read sequence information of index at the 3 'linker 